\title{
Comparison between surgery plus radiotherapy and radiotherapy alone in treating breast cancer patients with ipsilateral supraclavicular lymph node metastasis
}

\author{
Xian-Fu Sun $^{1 \# \wedge}$, Ying-Jie Wang ${ }^{2 \#}$, Tao Huang ${ }^{1 \wedge}$, Lian-Jie Niu ${ }^{1}$, Qiang Zhang ${ }^{1}, Z_{\text {Zhen-Zhen Liu }}{ }^{1}$ \\ ${ }^{1}$ Department of Breast Surgery, Affiliated Cancer Hospital of Zhengzhou University, Henan Cancer Hospital, Zhengzhou, China; ${ }^{2}$ Department of \\ Oncology, Affiliated Zhengzhou Cancer Hospital of Henan University, Zhengzhou Cancer Hospital, Zhengzhou, China \\ Contributions: (I) Conception and design: XF Sun, ZZ Liu; (II) Administrative support: XF Sun, ZZ Liu; (III) Provision of study materials or patients: \\ All authors; (IV) Collection and assembly of data: All authors; (V) Data analysis and interpretation: All authors; (VI) Manuscript writing: All authors; \\ (VII) Final approval of manuscript: All authors. \\ "These authors contributed equally to this work. \\ Correspondence to: Zhen-Zhen Liu. Department of Breast Surgery, Affiliated Cancer Hospital of Zhengzhou University, Henan Cancer Hospital, \\ Zhengzhou, China. Email: zlyyliuzhenzhen0800@zzu.edu.cn.
}

Background: Ipsilateral supraclavicular lymph node metastasis (ISLM) with breast cancer patients has always been a hard problem for breast surgery. It is generally believed that radiotherapy can benefit the survival of patients, but whether local surgical resection is needed or not is controversial. The study aims to compare the efficacy between supraclavicular lymph node (SLN) dissection combined with radiotherapy and radiotherapy alone in the treatment of breast cancer with ISLM.

Methods: A retrospective analysis was performed using 122 cases of breast cancer with ISLM but without distant metastasis. Among them, 14 cases were eliminated due to insufficient data. The 108 remaining cases were divided into 2 groups based on different treatment proposals for metastatic SLNs. The groups were dissection plus radiotherapy (surgery group), and simple radiotherapy (radiotherapy group).

Results: For the 108 patients, the overall 5-year disease-free survival (DFS) and overall survival (OS) rates were $30.6 \%$ and $67.8 \%$, respectively. In the surgery group, distant metastases occurred in 41 patients, and the 5-year DFS was $34.3 \%$; in the radiotherapy group, 18 patients had distant metastases, and the 5-year DFS was 26.1\%; the difference was not statistically significant $(\mathrm{P}>0.05)$. In the surgery group, 11 patients died, and the 5-year OS rate was $67.9 \%$; in the radiotherapy group, 6 patients died, and the 5 -year OS rate was $67.5 \%$; the difference was not statistically significant $(\mathrm{P}>0.05)$.

Conclusions: The dissection of SLN combined with radiotherapy and radiotherapy alone had similar effects on the survival rates in breast cancer patients with ISLM. The local control in the surgery group was better than that in the radiotherapy group. The status of estrogen receptors (ER) and the number of axillary lymph node metastases were independent influencing factors of DFS. The ER status is an independent factor affecting the OS rate of patients.

Keywords: Breast cancer; supraclavicular lymph node metastasis (ISLM); regional surgery; radiotherapy; estrogen receptor

Submitted Jul 28, 2020. Accepted for publication Oct 12, 2020.

doi: $10.21037 /$ gs-20-691

View this article at: http://dx.doi.org/10.21037/gs-20-691

\footnotetext{
^ ORCID: Xian-Fu Sun, 0000-0002-6512-080X; Tao Huang, 0000-0003-2607-8407.
} 


\section{Introduction}

Breast cancer is one of the most common malignant tumors in women. Ipsilateral supraclavicular lymph node metastasis (ISLM) of breast cancer is one of the predictors of poor prognosis (1). Wu et al. found that at the first visit, only $1-4 \%$ of breast cancer patients were diagnosed with ISLM without distant metastasis (2). The staging for ISLM has been the subject of continual change. In the 5 th edition of the American Joint Committee on Cancer (AJCC) staging system, ISLM was defined as a true distant metastasis (M1). However, current evidence has reported the survival of breast cancer patients with ISLM to be better than that of breast cancer patients with distant metastases, and that ISLM should be regarded as a regional lymph node metastasis rather than a distant metastasis (3). Therefore, according to the 2003 AJCC and Union for International Cancer Control (UICC) breast cancer staging guidelines, if there are no other distant metastases, the ISLM is divided into $\mathrm{N} 3 \mathrm{c}$ and clinical stage IIIC, which is considered to be a locally advanced breast cancer (4). Although the prognosis of breast cancer patients with ISLM is significantly better than that of actual M1 patients, comprehensive treatment should be provided for ISLM patients. Currently, there are no specific guidelines for the treatment of ISLM patients. In 1987, AJCC classified ISLM as M1, which was not cured by surgery. The main treatment of breast cancer was chemotherapy and radiotherapy. In 2001, Brito et al. (5) reported that 70 cases of breast cancer with isolated ISLM had a better prognosis than those with other distant metastasis after receiving chemotherapy, surgery and radiotherapy, and it was closer to stage III B breast cancer. Therefore, it was proposed that the breast cancer with isolated ISLM can be cured. The 6th AJCC staging guidelines further adjusted the staging of breast cancer, and classified the breast cancer with ISLM into stage III C, which was operable. The treatment gradually turned to the comprehensive treatment of primary surgery combined with radiotherapy and chemotherapy (6). Some researchers have described their experiences with surgery plus radiotherapy in treating the ISLM (7), while others have reported the effectiveness of radiotherapy alone in controlling supraclavicular metastatic sites; but whether there is survival difference between the different treatment methods remains unknown.

Therefore, the strategy of breast cancer with ISLM has always been controversial. At present, there is still a big controversy about whether such patients need supraclavicular lymph node dissection, and the national comprehensive cancer network (NCCN) guidelines do not explicitly recommend. It was found that the survival rate of patients with ISLM was similar to that of patients with stage III B, which was significantly better than that of patients with stage IV. The purpose of this study was to compare the efficacy of supraclavicular lymph node dissection combined with radiotherapy and radiotherapy alone in the conventional treatment mode of neoadjuvant chemotherapy combined with or without targeted therapy, so as to provide basis and new ideas for the choice in the treatment of this type breast cancer patients, and to benefit the survival of patients. We present the following article in accordance with the STROBE reporting checklist (available at http://dx.doi. org/10.21037/gs-20-691).

\section{Methods}

\section{Research object}

The Zhengzhou University Institutional Research Committee approved our study, and all patients provided signed informed consent before commencing treatment. The study was conducted in accordance with the Declaration of Helsinki (as revised in 2013). All methods were performed in accordance with $t /$ he relevant guidelines and regulations.

The medical records of primary breast cancer patients from January 2010 to January 2019 were retrospectively reviewed.

The inclusion criteria were as follows: age 2172 years; diagnosis of ISLM by preoperative coarse needle biopsy; ISLM treated with surgery plus radiotherapy or radiotherapy alone; no distant metastasis at the first visit; no severe disease of the center of gravity, liver, kidney and hematopoietic system; ECOG score of 0-2; complete case data. Exclusion criteria: bilateral breast cancer patients; distant metastasis in the first visit; poor general condition, unable to tolerate surgery and chemotherapy; incomplete case data.

A total of 108 patients were enrolled, and were divided into 2 groups based on different treatment for supraclavicular lymph nodes (SLNs): dissection plus radiotherapy group $(\mathrm{n}=84)$, and radical radiotherapy group $(\mathrm{n}=24)$. The 2 groups were similarly distributed regarding age, sex, tumor stage (TNM), and other clinical-pathologic variables, as shown in Table 1 (all $\mathrm{P}>0.05$ ). 
Table 1 Comparison of clinical characteristic parameters between surgery group and radiotherapy group

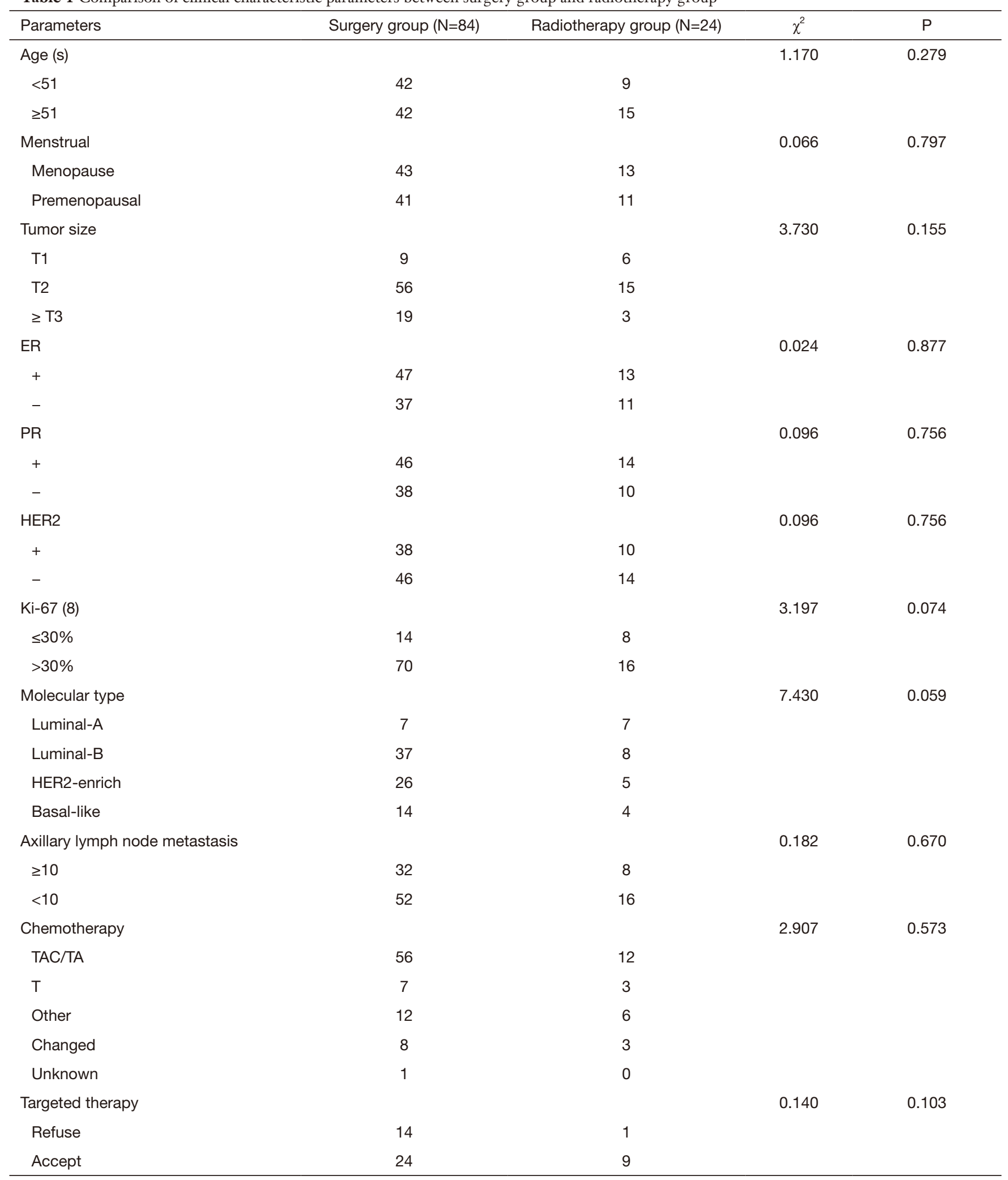

ER, estrogen receptor; PR, progesterone receptor; HER2, human epidermal growth factor receptor 2. 


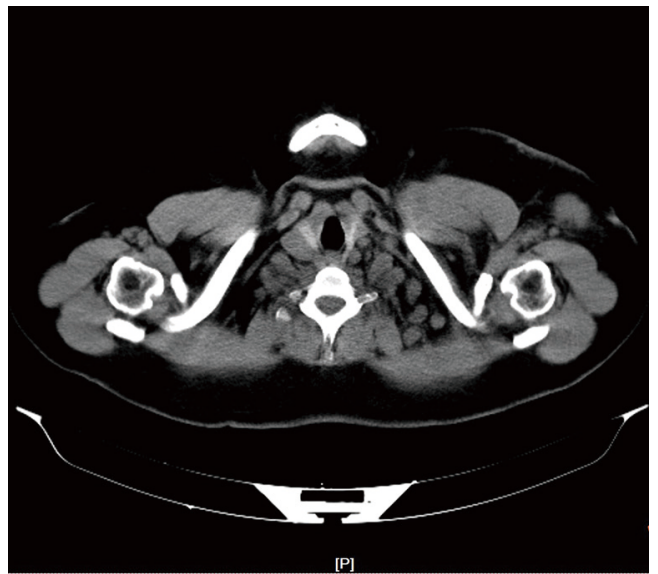

Figure 1 Supraclavicular lymph nodes metastasis in CT.

\section{Research method}

The participants routinely underwent bone scan, ultrasonography, CT (Figure 1), MRI, and/or PETCT prior to definite treatment. All patients in this study received 4-6 cycles of neoadjuvant chemotherapy mainly anthracycline and docetaxel after pathological puncture confirming ISLM. All patients underwent radical or modified radical mastectomy. Lymph nodes with axillary I, II, and III levels were dissected. Cervical III, IV, and V lymph nodes were dissected in the supraclavicular dissection group. After operation and chemotherapy, the patients received combined extracorporeal radiotherapy of the chest wall, regional lymphatic vessels, and supraclavicular area. The dose of the surgery group was $50 \mathrm{~Gy}, 25$ times for 5 weeks; the dose of radiotherapy group was $60 \mathrm{~Gy}$, 25 times for 5 weeks. All patients with positive hormone receptors received further hormone therapy. There were 15 human epidermal growth factor receptor 2 (HER2)positive patients (14 cases in operation plus radiotherapy group, and 1 case in the radiotherapy group) who refused to receive targeted therapy because of their economic status, and the rest received targeted therapy regularly (trastuzumab was administered for 1 year, initial dose was $8 \mathrm{mg} / \mathrm{kg}$, subsequent doses were $6 \mathrm{mg} / \mathrm{kg}$, with 3 weeks constituting a course of treatment).

Follow-up was performed from the first day following diagnosis. All 108 patients were followed up by the outpatient clinic, telephone, and case consultation. The follow-up period was 10-96 months, and the median follow-up time was 75 months. The follow-up period ended on December 31, 2019.
Local recurrence refers to pathological recurrence in the ipsilateral chest wall, supraclavicular and subclavian fossa, and axillary or internal mammary region. Distant metastasis was confirmed by 2 types of medical imaging (and pathological examination if necessary) to confirm that the cancer had spread distal to the local recurrence site. Disease-free survival (DFS) is defined as the time from surgery to recurrence or death due to disease progression. The overall survival time (OS) is defined as the time from the diagnosis of breast cancer to death from whatever cause, regardless of whether the death is associated with breast cancer or not.

\section{Statistical analysis}

The statistical analysis was carried out by SPSS 24.0 software (SPSS Inc., IBM, Chicago, IL, USA); the difference between qualitative data was analyzed by the chi-squared $\left(\chi^{2}\right)$ and Fisher's exact probability test; the survival rate was compared by the Kaplan-Meier method; and the survival curve was compared by log-rank test. The significance of the test level was deemed to be $\alpha=0.05$.

\section{Results}

Comparison of 5-year OS and DFS between the two groups

Amongst the 108 patients, the overall 5-year DFS and OS rates were $30.6 \%$ and $67.8 \%$, respectively. In the surgery group, distant metastasis occurred in 41 patients, and the 5 -year DFS was $34.3 \%$; in the radiotherapy group, 18 patients had distant metastases, and the 5 -year DFS was $26.1 \%$; the difference was not statistically significant (Figure 2). In the surgery group, 11 patients died, and the 5 -year OS rate was $67.9 \%$; in the radiotherapy group, 6 patients died, and the 5 -year OS rate was $67.5 \%$; the difference was not statistically significant (Figure 3).

\section{Cox regression analysis of 5-year DFS}

Univariate analysis showed that estrogen receptor (ER) status $(\mathrm{P}=0.003)$, and the number of axillary lymph node metastases $(\mathrm{P}=0.008)$ were correlated with the 5 -year DFS rate, while other clinicopathological features and treatment methods had no effect on the 5 -year DFS rate. Cox regression analysis showed that ER status (95\% CI: $1.337-3.889, \mathrm{P}=0.002)$ and the number of axillary lymph 


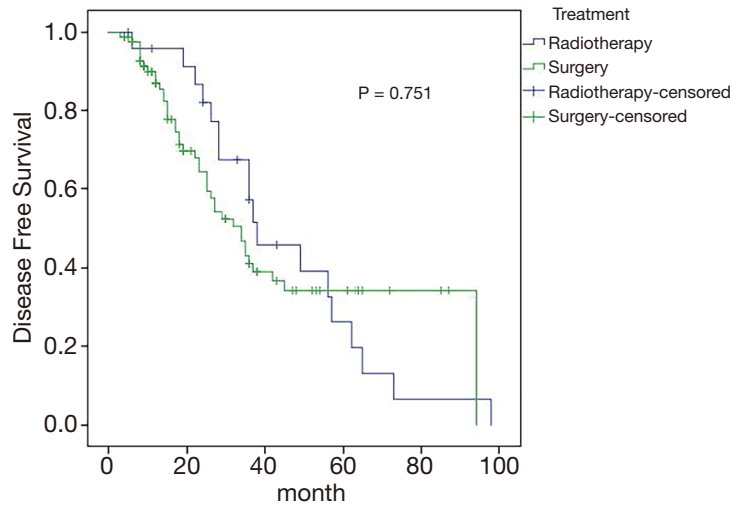

Figure 2 Estimated overall survival curves for DFS comparing the surgery and radiotherapy. DFS, disease-free survival.

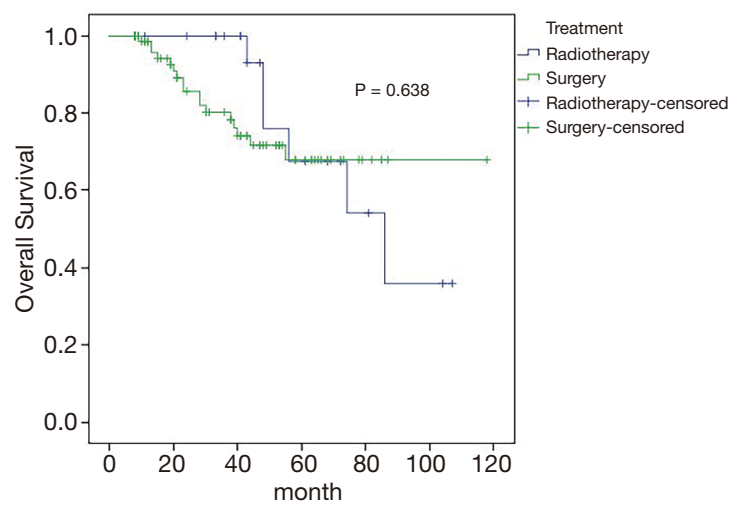

Figure 3 Estimated overall survival curves for OS comparing the surgery and radiotherapy. OS, overall survival.

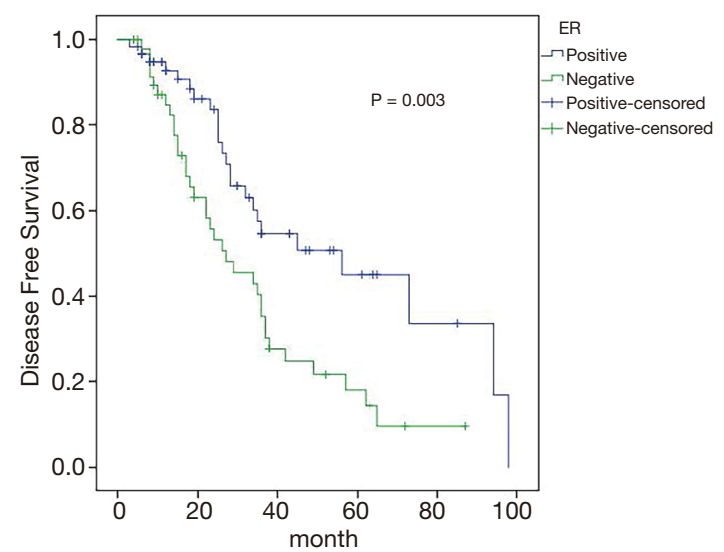

Figure 4 Effect of ER status on DFS. ER, estrogen receptor; DFS, disease-free survival.

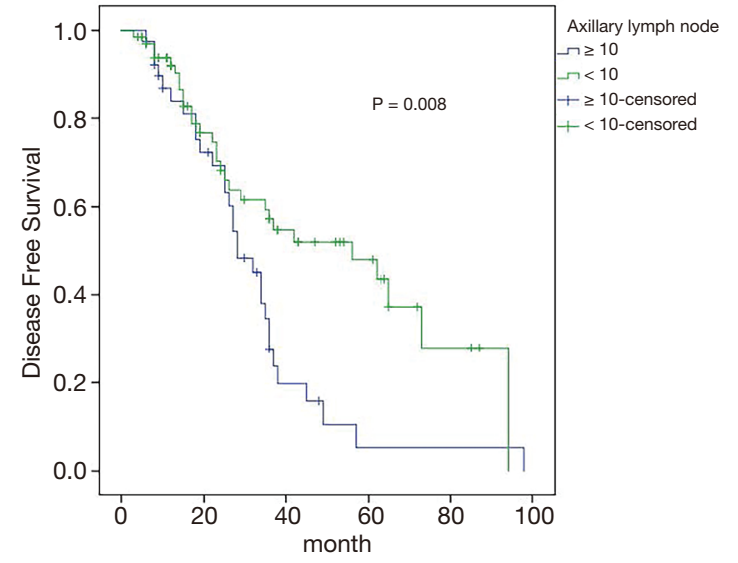

Figure 5 Effect of axillary lymph node metastasis number on DFS rate. DFS, disease-free survival.

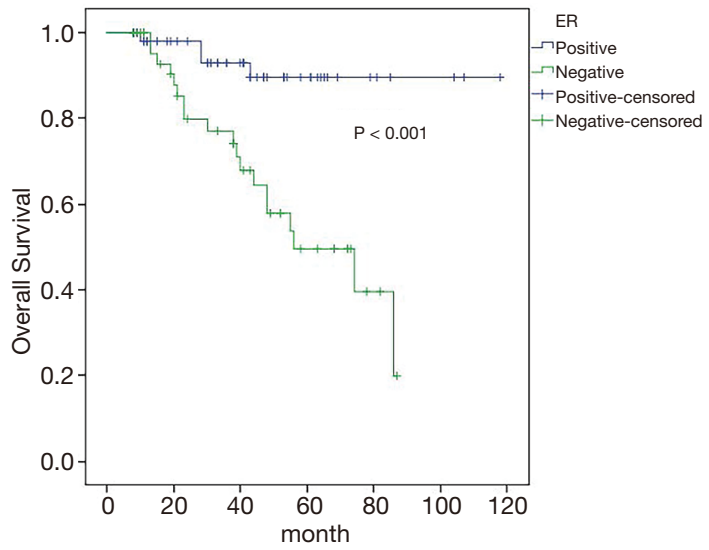

Figure 6 Effect of ER status on OS. ER, estrogen receptor; OS, overall survival.

node metastases (95\% CI: 1.259-3.627, $\mathrm{P}=0.005$ ) were independent influencing factors of 5 -year DFS in patients with ISLM breast cancer (Figures 4,5).

\section{Cox regression analysis of 5-year OS}

Univariate analysis showed that ER status $(\mathrm{P}<0.001)$, progesterone receptor $(\mathrm{PR})$ status $(\mathrm{P}=0.007)$, and the molecular typing $(\mathrm{P}=0.002)$ were associated with the 5 -year OS rate. Cox regression analysis showed that ER status (95\% CI: 1.100-18.288) was an independent influencing factor of 5-year OS (Figure 6). 


\section{Discussion}

The incidence of breast cancer with ISLM but without distant metastasis is about $1-4 \%$ (9), and the prognosis is poor, with a published 5-year OS of 5-34\% (10-12). This type of cancer has been previously classified as M1. Recently, Bertero et al. (13) reported that the 5-year OS of synchronous ISLM was $41 \%$ after multimode treatment, which was significantly better than that of women with distant metastases. This led to revision of the AJCC staging system to re-classify ISLM as $\mathrm{pN} 3$ phase IIIC disease (14). Chang et al. (15) firstly analyzed the clinical value of SLN dissection and radiotherapy in treating patients with ISLM; in the SLN dissection and radiotherapy groups, the 3-year DFS was $46.2 \%$ and $31.5 \%$, respectively, and the 5 -year OS was $46.2 \%$ and $37.5 \%$, respectively, but no significant difference. In the current study, we also noted there was no additional survival benefit associated with surgery. However, Tezuka et al. (16) found that local surgical treatment of ISLM can lead to a good prognosis. In a similar study, local surgery was also shown to be a prognostic factor for OS (17). These findings would suggest that, when radiotherapy could not be provided to patients to maximize local area control, local surgical treatment can be considered. Our 5 -year OS rate was higher than that in other studies, which may be related to the improvement of systemic therapy and individualization of treatment.

In the latest international staging systems of AJCC and UICC for breast cancer, ISLM has been divided into locally advanced $\mathrm{N} 3 \mathrm{c}$, but the specific location of metastasis has not been clearly identified. Sesterhenn et al. (18) drew lessons from the anatomical localization of cervical lymph nodes in head and neck surgery in the study of 12 cases of ISLM, and pointed out that the supraclavicular region is relative to the cervical IV (inferior jugular vein) and $\mathrm{V}$ region (posterior cervical triangle). The higher position of III and II (upper and middle segment of jugular vein) did not belong to the drainage area of a conventional mammary gland. This classification is significantly better than the classification of SLNs. All lymph node metastases higher than the superior jugular vein should be allocated into M1, which provides a reference for the clinical dissection of SLNs (19). However, because of the special anatomical location of SLNs, radical lymph node dissection is difficult in breast cancer patients with ISLM (20). Therefore, radiotherapy has become the main local treatment of ISLM. The findings of Fan et al. (21) suggested that there was no significant difference in local recurrence rate, supraclavicular recurrence rate, DFS rate, and OS rate 3-5 years after radical radiotherapy. Perloff et al. (22) compared the effects of simple surgery and radiotherapy after neoadjuvant chemotherapy for locally advanced breast cancer. The results showed that there was no significant difference in the local control rate between operation alone and radiotherapy alone.

In our study, the 5 -year DFS rates of the surgery group and radiotherapy group were $34.3 \%$ and $26.1 \%$, respectively, and the 5-year OS rates of the surgery group and radiotherapy group were $67.9 \%$ and $67.5 \%$, respectively, and the difference was not statistically significant $(\mathrm{P}>0.05)$. There is no difference in the efficacy of SLN dissection or radiotherapy for patients with ISLM breast cancer. During the follow-up period, a total of 59 patients had recurrence and metastasis, 34 cases (40.5\%) in the surgery group, and 11 cases $(45.8 \%)$ in the radiotherapy group, the difference was not statistically significant $(\mathrm{P}>0.05)$. Local recurrence occurred in 15 cases $(17.9 \%)$ in the surgery group, and 15 cases $(62.5 \%)$ in the radiotherapy group $(\mathrm{P}>0.001)$. Recurrence and metastasis occurred in 8 cases $(9.5 \%)$ in the surgery group, and 8 cases $(33.3 \%)$ in the radiotherapy group $(\mathrm{P}>0.05)$. In the surgery group, 17 cases died (20.2\%), in the radiotherapy group, 6 cases died (25.0\%), and the difference was not statistically significant $(\mathrm{P}=0.615)$. We believe that although the increase of regional surgery and radiotherapy alone have no effect on the OS and DFS, the primary supraclavicular metastasis still has the potential for metastasis and recurrence. This possibility diminishes patient quality of life, and delivers physical, psychological, and economic pressure, so increasing SLN dissection may be an effective means of local control.

Axillary lymph node metastasis is an independent prognostic factor in patients with breast cancer, lymph nodes in the primary tumor region are first metastasized to the sentinel lymph nodes through the lymphatic vessels in a predictable order, and then to the distal lymph nodes (23). Therefore, when there are more axillary lymph node metastases, we think there is a greater possibility of lymph node metastasis to the next station. In our study, the 5 -year DFS rate of patients with an axillary lymph node positive number $\geq 10$ was $5.3 \%$, while the 5 -year DFS rate of patients with an axillary lymph node positive number $<10$ was $47.8 \%$, and the difference was statistically significant. It is concluded that the number of axillary lymph node metastases is an independent factor affecting 5-year DFS in patients with ISLM breast cancer.

Status and expression of ER, progesterone receptor $(\mathrm{PR})$, and HER2 are very important for the treatment 
and prognosis of the transformation of ER, progesterone receptor $(\mathrm{PR})$, and $\mathrm{HER} 2$, because they may affect treatment choice and efficacy $(24,25)$. The results of univariate prognostic analysis in our study showed that the 5 -year DFS rate of ER-positive patients was 43.6\%, and the 5-year DFS rate of ER-negative patients was $18.5 \%$, and the difference was statistically significant $(\mathrm{P}=0.003)$; ERpositive patients 5 -year OS rate was $89.4 \%$, and that of ERnegative patients was $49.5 \%$, the difference was statistically significant $(\mathrm{P}<0.01)$. Cox regression analysis showed that ER status was an independent factor affecting DFS and OS. Negative ER is associated with poor prognosis, so administering more active treatment may be necessary for these patients.

In summary, although ISLM is considered to be a curable local disease, comprehensive treatment should be provided to ISLM patients, including surgery, radiotherapy, endocrine therapy, targeted therapy, among others. Because the local control in the surgery group was better than that in the radiotherapy group, we conclude that the increasing local surgery can benefit patient survival rates. The status of ER and the number of axillary lymph node metastases were independent influencing factors of DFS. The ER status is an independent factor affecting the OS rate of patients.

\section{Acknowledgments}

Funding: None.

\section{Footnote}

Reporting Checklist: The authors have completed the STROBE reporting checklist. Available at http://dx.doi. org/10.21037/gs-20-691

Data Sharing Statement: Available at http://dx.doi. org/10.21037/gs-20-691

Conflicts of Interest: All authors have completed the ICMJE uniform disclosure form (available at http://dx.doi. org/10.21037/gs-20-691). The authors have no conflicts of interest to declare.

Ethical Statement: The authors are accountable for all aspects of the work in ensuring that questions related to the accuracy or integrity of any part of the work are appropriately investigated and resolved. The Zhengzhou University Institutional Research Committee approved our study, and all patients provided signed informed consent before commencing treatment. The study was conducted in accordance with the Declaration of Helsinki (as revised in 2013).

Open Access Statement: This is an Open Access article distributed in accordance with the Creative Commons Attribution-NonCommercial-NoDerivs 4.0 International License (CC BY-NC-ND 4.0), which permits the noncommercial replication and distribution of the article with the strict proviso that no changes or edits are made and the original work is properly cited (including links to both the formal publication through the relevant DOI and the license). See: https://creativecommons.org/licenses/by-nc-nd/4.0/.

\section{References}

1. Dellapasqua S, Bagnardi V, Balduzzi A, et al. Outcomes of patients with breast cancer who present with ipsilateral supraclavicular or internal mammary lymph node metastases. Clin Breast Cancer 2014;14:53-60.

2. Wu SG, Sun JY, Zhou J, et al. The value of radiotherapy in breast cancer patients with isolated ipsilateral supraclavicular lymph node metastasis without distant metastases at diagnosis: a retrospective analysis of Chinese patients. Onco Targets Ther 2014;7:281-8.

3. Grotenhuis BA, Klem TM, Vrijland WW. Treatment outcome in breast cancer patients with ipsilateral supraclavicular lymph node metastasis at time of diagnosis: a review of the literature. Eur J Surg Oncol 2013;39:207-12.

4. Singletary SE. Revision of the American Joint Committee on Cancer Staging System for Breast Cancer. J Clin Oncol 2002;20:3628-36.

5. Brito RA, Valero V, Buzdar AU, et al. Long-term results of combined-modality therapy for locally advanced breast cancer with ipsilateral supraclavicular metastases: The University of Texas M. D. Anderson Cancer Center experience. J Clin Oncol 2001;19:628-33.

6. Yoon WS, Rim CH, Yang DS, et al. Long-term outcomes of immediate autologous breast reconstruction after definite adjuvant therapy in intermediate and locally advanced breast cancer. Chronic Diseases and Translational Medicine 2016;1:236-42.

7. Winters ZE, Bernaudo L. Evaluating the current evidence to support therapeutic mammoplasty or breast-conserving surgery as an alternative to mastectomy in the treatment of multifocal and multicentric breast cancers. Gland Surg 
2018;7:525-35.

8. Cheang MCU, Chia SK, David V, et al. Ki67 Index, HER2 Status, and Prognosis of Patients with Luminal B Breast Cancer. JNCI 2009;101:736-50.

9. Olivotto IA. Long-Term Survival of Patients With Supraclavicular Metastases at Diagnosis of Breast Cancer. J Clin Oncol 2003;21:851-4.

10. Fodor J, Toth J, Major T, et al. Incidence and time of occurrence of regional recurrence in stage I-II breast cancer: Value of adjuvant irradiation. Int J Radiat Oncol Biol Phys 1999;44:281-7.

11. McKinna F, Gothard L, Ashley S, et al. Lymphatic relapse in women with early breast cancer: a difficult management problem. Eur J Cancer 1999;35:1065.

12. Pedersen AN, Møller S, Steffensen KD, et al. Supraclavicular recurrence after early breast cancer: a curable condition? Breast Cancer Res Treat 2011;125:815-22.

13. Bertero L, Massa F, Metovic J, et al. Eighth Edition of the UICC Classification of Malignant Tumours: an overview of the changes in the pathological TNM classification criteria-What has changed and why? Virchows Arch 2018;472:519-31.

14. Pergolizzi S, Adamo V, Russi E, et al. Prospective multicenter study of combined treatment with chemotherapy and radiotherapy in breast cancer women with the rare clinical scenario of ipsilateral supraclavicular node recurrence without distant metastases. Int J Radiat Oncol Biol Phys 2006;65:25-32.

15. Chang XZ, Yin J, Sun J, et al. A retrospective study of different local treatments in breast cancer patients with synchronous ipsilateral supraclavicular lymph node metastasis. J Cancer Res Ther 2013;9:S158-61.

16. Tezuka K, Dan N, Tendo M, et al. A case of breast cancer with postoperative metastasis to the supraclavicular lymph nodes-recurrence-free survival achieved by surgical excision following chemotherapy. Gan To Kagaku Ryoho 2011;38:1345-7.

Cite this article as: Sun XF, Wang YJ, Huang T, Niu LJ, Zhang Q, Liu ZZ. Comparison between surgery plus radiotherapy and radiotherapy alone in treating breast cancer patients with ipsilateral supraclavicular lymph node metastasis. Gland Surg 2020;9(5):1513-1520. doi: 10.21037/gs-20-691
17. Chen SC, Chang HK, Lin YC, et al. Prognosis of Breast Cancer After Supraclavicular Lymph Node Metastasis: Not a Distant Metastasis. Ann Surg Oncol 2006;13:1457-65.

18. Heidenreich A, Sesterhenn IA, Mostofi FK, et al. Prognostic risk factors that identify patients with clinical stage I nonseminomatous germ cell tumors at low risk and high risk for metastasis. Cancer 1998;83:1002-11.

19. Zhang Y, Liu Y. Treatment of breast cancer with ipsilateral supraclavicular lymph node metastasis. Mod Oncol Med 2017;25:2167-70.

20. Aldridge T, Kusanale A, Colbert S, et al. Supraclavicular metastases from distant primaries: what is the role of the head and neck surgeon? Br J Oral Maxillofac Surg 2013;51:288-93.

21. Fan Y, Xu B, Liao Y, et al. A retrospective study of metachronous and synchronous ipsilateral supraclavicular lymph node metastases in breast cancer patients. Breast 2010;19:365-9.

22. Perloff M, Lesnick GJ, Korzun A, et al. Combination chemotherapy with mastectomy or radiotherapy for stage III breast carcinoma: a Cancer and Leukemia Group B study. J Clin Oncol 1988;6:261-9.

23. Camp RL, Rimm EB, Rimm DL, et al. A high number of tumor free axillary lymph nodes from patients with lymph node negative breast carcinoma is associated with poor outcome. Cancer 2000;88:108-13.

24. Nauroth A, Kalder M, Rössler M, et al. Conversion of hormone and HER-2 receptor in metachronous neck metastases from breast carcinoma. J Cancer Res Clin Oncol 2017;143:1811-4.

25. Vogel C, Malter W, Morgenstern B, et al. The Role of Previous Therapies and Sites of Metastasis as Influencing Factors on Discordance of ER, PR and HER2 Status Between Primary and Metastasized Breast Cancer. Anticancer Res 2019;39:2647.

(English Language Editor: J. Jones) 